\title{
Erratum
}

\section{Erratum to "An Efficient Code-Based Threshold Ring Signature Scheme with a Leader-Participant Model"}

\author{
Guomin Zhou, ${ }^{1}$ Peng Zeng, ${ }^{2}$ Xiaohui Yuan, ${ }^{3,4}$ \\ Siyuan Chen, ${ }^{2}$ and Kim-Kwang Raymond $\mathrm{Choo}^{5}$ \\ ${ }^{1}$ Department of Computer and Information Technology, Zhejiang Police College, Hangzhou, Zhejiang Province, China \\ ${ }^{2}$ Shanghai Key Laboratory of Trustworthy Computing, East China Normal University, Shanghai, China \\ ${ }^{3}$ Department of Computer Science and Engineering, University of North Texas, Denton, TX 76203, USA \\ ${ }^{4}$ College of Information Engineering, China University of Geosciences, Wuhan, China \\ ${ }^{5}$ Department of Information Systems and Cyber Security, University of Texas at San Antonio, San Antonio, TX 78249, USA \\ Correspondence should be addressed to Peng Zeng; pzeng@sei.ecnu.edu.cn and Xiaohui Yuan; xiaohui.yuan@unt.edu \\ Received 30 August 2017; Accepted 2 October 2017; Published 16 October 2017 \\ Copyright (c) 2017 Guomin Zhou et al. This is an open access article distributed under the Creative Commons Attribution License, \\ which permits unrestricted use, distribution, and reproduction in any medium, provided the original work is properly cited.
}

In the article titled "An Efficient Code-Based Threshold Ring Signature Scheme with a Leader-Participant Model” [1], Dr. Peng Zeng should be listed as a corresponding author.

\section{References}

[1] G. Zhou, P. Zeng, X. Yuan, S. Chen, and K. R. Choo, "An efficient code-based threshold ring signature scheme with a leaderparticipant model," Security and Communication Networks, vol. 2017, Article ID 1915239, 7 pages, 2017. 


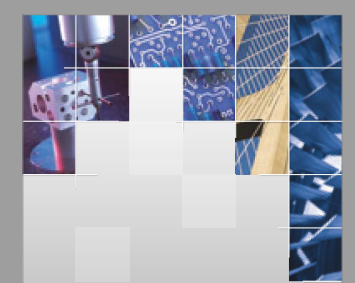

\section{Enfincering}
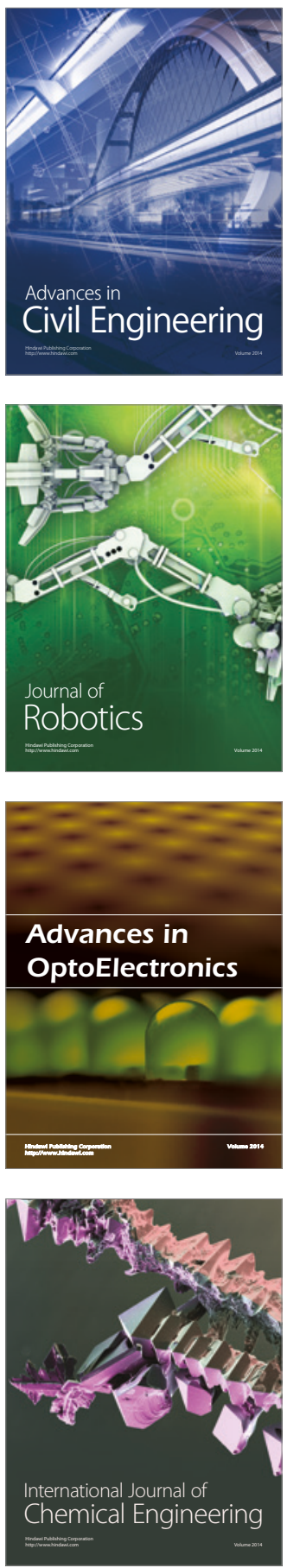

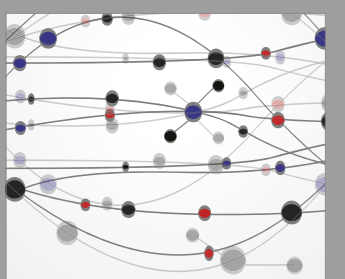

The Scientific World Journal

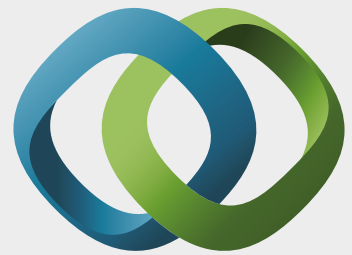

\section{Hindawi}

Submit your manuscripts at

https://www.hindawi.com
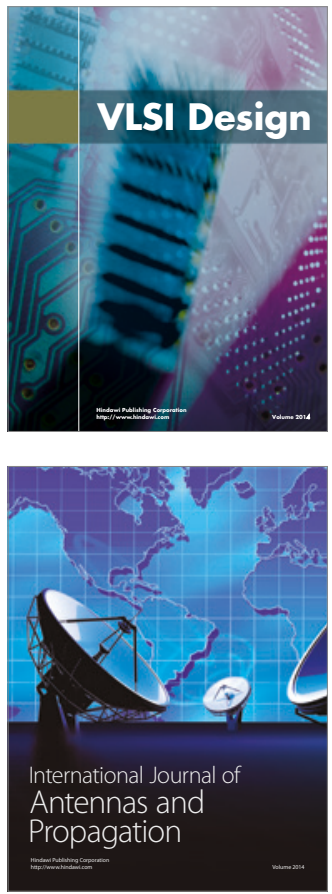

\section{Rotating}

Machinery
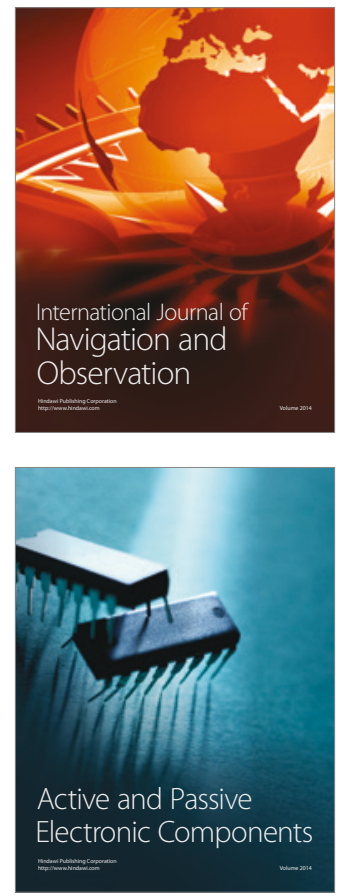
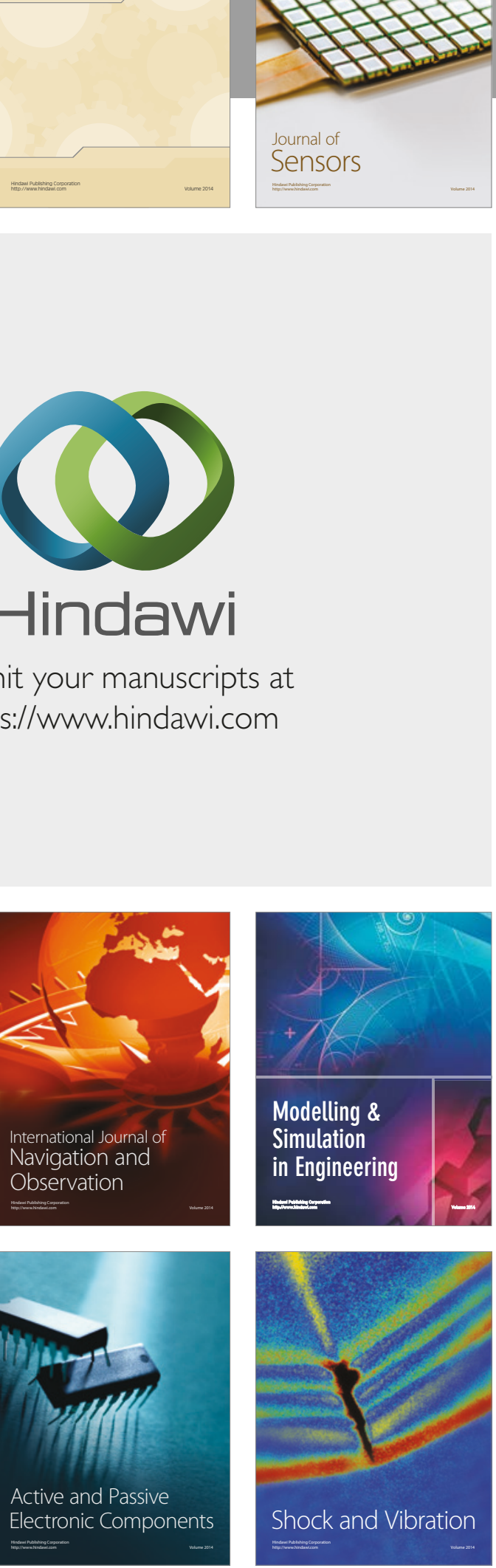
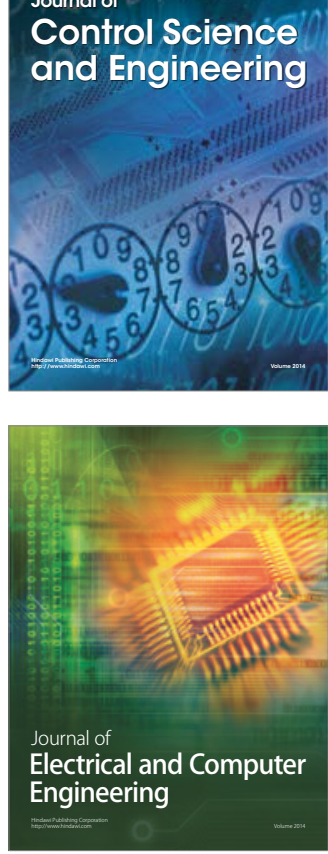

Distributed

Journal of

Control Science

and Engineering
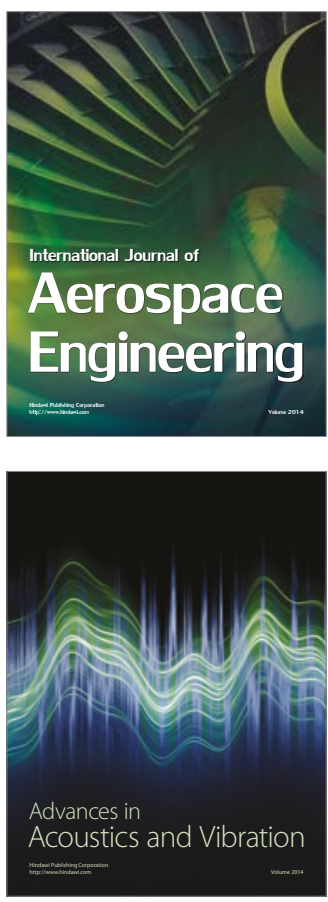

Sensor Networks 